\title{
Endometrial Receptivity Markers in Patients with Ovulatory Polycystic Ovary Syndrome, Endometrioma and Unexplained Subfertility: A Prospective Comparative Study
}

\author{
Emine Demirel $^{1 *}$, Fulya Oguz ${ }^{1}$, Melike Demir Caltekin ${ }^{1}$, Mustafa Sengul ${ }^{1}$, Burak Yucel ${ }^{2}$ and Sefa Kelekci ${ }^{1}$ \\ ${ }^{1}$ Izmir Katip Celebi University, Atatürk Training and Research Hospital, Faculty of Medicine, Department of Obstetrics and Gynecology, Izmir, \\ Turkey \\ ${ }^{2}$ Istanbul Kanuni Sultan Süleyman Education and Research Hospital, İstanbul, Turkey \\ * Corresponding Author: Emine Demirel, M.D., Izmir Katip Celebi University Faculty of Medicine, Department of Obstetrics and \\ Gynecology, Izmir, Turkey. E-mail: er_em.dr@hotmail.com. Phone: +90232 243 43 43, Fax: +90 2322431530 \\ Received Date: October 27, 2019; Accepted Date: November 1, 2019; Published Date: November 11 , 2019 \\ Citation: Emine Demirel, Fulya Oguz, Melike Demir Caltekin, Mustafa Sengul, Burak Yucel and Sefa Kelekci.(2019) Endometrial receptivity \\ markers in patients with ovulatory polycystic ovary syndrome, endometrioma and unexplained subfertility: A prospective comparative study. \\ Obstetrics Gynecology and Reproductive Sciences, 3(2): DOI: 10.31579/2578-8965/025 \\ Copyright: (C2019. Emine Demirel. This is an open-access article distributed under the termsof the Creative Commons Attribution License, \\ which permits unrestricted use, distribution, and reproduction in any medium, provided the original author and source are credited.

\section{Abstract} \\ Many molecular markers have been identified in different stages of the luteal phase those play roles in the implantation. Our \\ aim was to compare the levels of insulin-like growth factor binding protein 1 (IGFBP-1), Osteopontin (OPN) and \\ prostaglandin E2 (PGE2) in endometrial washing liquid between women with ovulatory polycystic ovary syndrome (PCOS), \\ endometrioma and unexplained subfertility, compared with ovulatory women. The study groups were formed by women \\ with ovulatory PCOS $(n=24)$, endometrioma $(n=17)$ and unexplained subfertility $(n=25)$. The control group consisted of \\ fertile women $(n=18)$. There were no significant differences in terms of the values of IGFBP1, PGE2 and OPN among \\ groups. There was a statistically significant difference between ovulatory PCOS group and control group in terms of PGE2 \\ levels ( $\mathrm{p}=0.002$ ). High PGE2 might be a marker for poor endometrial receptivity. We supposed that the down-regulation of \\ PGE2 may facilitate decidualization and improve pregnancy rate in ovulatory PCOS.
}

Key words: Endometrial receptivity; insulin-like growth factor binding protein 1; osteopontin, prostaglandin E2; polycystic ovary syndrome; unexplained subfertility.

\section{Impact Statement}

- What is already known on this subject: Endometrial receptivity markers are expressed in the implantation window for successful embryo implantation. Insulin-like growth factor binding protein 1 (IGFBP-1), prostaglandin E2 (PGE2) and osteopontin (OPN) have significant roles in endometrial function and implantation. These would be potential biomarkers of endometrial receptivity.

- What The Results Of This Study Add: High PGE2 levels in women with ovulatory polycystic ovary syndrome (PCOS) may contribute to endometrial dysfunction and subfertility according to results of our study.

- What Are The Implications Of These Findings Clinical Practice And/Or Further Research: PGE2 may be an indicator of reduced endometrial receptivity that might be responsible for the low pregnancy rates in women with ovulatory PCOS.

\section{Introduction}

A successful embryo implantation requires a receptive endometrium, a live embryo and harmonious signalization between them. After the contact between cytokines, growth factors, receptors and blastocyst, junctional complexes are formed. Then blastocyst adhesion and invasion starts via adhesion molecules such as integrins and selectins [1].
The implantation window is defined as that period when the uterus is receptive for implantation of the free-lying blastocyst. Some important growth changes occur during implantation window. Recently, many molecular markers have been identified in different stages of the luteal phase those play roles in the implantation. These endometrial receptivity markers are expressed in the implantation window for successful implantation [2-5].

Insulin-like growth factor binding protein 1 (IGFBP-1) has an important role in growth, development and apoptosis in endometrium. It is secreted from the ovarian stroma depending on the phase of the menstrual cycle and has a role in the decidual differentiation of stroma as a specific decidualization marker [6]. Recent studies have shown that androgen precursor dehydroepiandrosterone (DHEA) and the distal upstream region of insulin-like growth factor-binding protein-1 can enhances expression of IGFBP-1 in endometrial stromal cells during decidualization and may improve pregnancy rates in natural or assisted reproductive cycles $[7,8]$. Osteopontin (OPN) presents in the endometrium in a coordinated manner throughout the menstrual cycle of fertile women and is expressed at maximum levels during the implantation window $[9,10]$. It was suggested that this protein has complementary roles in endometrial function and implantation and that OPN and its receptor, $\alpha v \beta 3$ integrin, complex might be formed to support embryo attachment [10-14]. Furthermore, Wang et al [15] demonstrated that level of OPN is significantly repressed in the failed group when compared with successful pregnancy group in in vitro 
fertilization (IVF) cycles and therefore evidence supporting the fact that OPN is involved in decidualization and pregnancy success. Prostaglandins are a group of bioactive lipid products formed as a result of arachidonic acid metabolism. Vilella et al [16] suggest that prostaglandin E2 (PGE2) concentrations 24 hours prior to embryo transfer are potential noninvasive biomarkers of endometrial receptivity.

The aim of this prospective cross-sectional study was to compare the levels of IGFBP-1, osteopontin and PGE2 in the late luteal phase endometrial washing fluids of healthy, fertile women and patients with ovulatory PCOS, endometrioma and unexplained subfertility.

\section{Materials and Methods}

This study was conducted between January 2013 and June 2013 in subfertility unit of the Department of Obstetrics and Gynecology, School of Medicine, Izmir Katip Celebi University, Izmir, Turkey. The unit is a tertiary center in the west of Turkey that treats referral patients from the region. The study design was in accordance with the ethical standards of the Helsinki declaration and was approved by the Institutional Review Board of İzmir Katip Celebi University School of Medicine (17.05.2012/22). Written informed consent was taken from all volunteers.

One hundred and twelve women between 20 and 40 years' old were included. Patients were recruited who admitted to subfertility outpatient clinic with a sequential manner because of seeking fertility. Control group was selected among age matched woman who admitted to family planning unit for requesting contraception. Inclusion criteria of study group were ovulatory PCOS, subfertil woman with endometrioma and unexplained subfertility. The control group consisted of fertile women with no gynecologic disorder, not using an intrauterine device or hormonal contraception. Exclusion criteria were anovulatuar woman with PCOS and control group, smoking, pelvic infection, endometrial pathology (endometrial polyp, submucosal myoma etc.) during the endometrial fluid sampling.

Main outcome measure was compare IGFBP-1, PGE2, OPN levels during implantation window in ovulatory PCOS, endometrioma, unexplained subfertility and fertile ovulatory women.
The endometrial fluid sampling was performed after ovulation was confirmed. $0.154 \mathrm{~mol} / \mathrm{L}$ sodium chloride was administered via a thin cannula. A total of $10 \mathrm{~mL}$ ( $2 \mathrm{~mL}$ per administration for 5 times) endometrial fluid sample was collected for each patient. The $1 \mathrm{ml}$ of the aspirate was poured into a standard $1.5 \mathrm{ml}$ micro test tube (Eppendorf, Hamburg, Germany), was frozen at $-20^{\circ} \mathrm{C}$ and was stored in a deep freezer at $-80^{\circ} \mathrm{C}$ until biochemical analyses were performed. After the endometrial fluid samples were collected from the patients, IGFBP-1, osteopontin and PGE2 levels were studied by using East biopharm branded (Hangzhou East biopharm Co., Ltd./China) Elisa kits (PGE2 lot: 20130924, OPN lot: 20130924, IGFBP-1 lot: 20130924, PGE2 Cat. No: CK-E10702, OPN Cat. No: CK-E10857, IGFBP-1 Cat. No: CK-E10159) with the Biotec branded Elisa device.

For the statistical analysis, the SPSS (version15.0, 2006; SPSS Inc., Chicago, IL, USA) program was used. The distribution of the data was controlled by Shapiro-Wilks and Levene's tests. As the fundamental hypothesis of parametric statistics was not met, instead of parametric MANOVA, use of non-parametric tests was considered appropriate. Hence, for both variables Kruskal-Wallis tests were performed, four groups were tested in the same hypothesis and paired comparisons were made between the groups via Mann-Whitney U tests as follow-up tests in case statistically significant differences were achieved. $\mathrm{P}<0.05$ was accepted as statistically significant.

\section{Results}

A total of 120 women were included. The study groups were formed by patients with ovulatory PCOS $(n=38)$, endometrioma $(n=19)$ and unexplained subfertility $(\mathrm{n}=27)$. The control group consisted of fertile women $(n=28)$ with no gynecologic disorder, not using an intrauterine device or hormonal contraception. Twenty-eight women $(23.3 \%)$ with anovulation (a serum progesterone level of $<3 \mathrm{ng} / \mathrm{dL}$ on the 21 st day of menstruation) in both study and control groups were excluded. Twentyfour patients with ovulatory PCOS, 25 with unexplained subfertility, 17 with endometrioma and 18 healthy fertile ovulatory women were analyzed. All groups were similar in terms of demographically except for gravidity and parity [Table 1].

\begin{tabular}{|c|c|c|c|c|c|}
\hline & $\begin{array}{c}\text { PCOS } \\
(\mathbf{n = 2 4})\end{array}$ & $\begin{array}{c}\text { US } \\
(\mathbf{n = 2 5})\end{array}$ & $\begin{array}{c}\text { Endo } \\
(\mathbf{n = 1 7})\end{array}$ & $\begin{array}{c}\text { Control } \\
(\mathbf{n = 1 8})\end{array}$ & P-value* \\
\hline Age (years) & $29.87 \pm 5.61$ & $29.05 \pm 4.81$ & $33.05 \pm 6.68$ & $33.55 \pm 5.90$ & 0.013 \\
\hline BMI (kg/m $)$ & $28.67 \pm 7.93$ & $24.25 \pm 3.61$ & $24.11 \pm 3.56$ & $25.16 \pm 2.68$ & 0.209 \\
\hline $\begin{array}{c}\text { Gravida } \\
\text { Median(min-max) }\end{array}$ & $1(0-1)$ & $1(0-1)$ & $1(0-2)$ & $2(1-6)$ & 0.000 \\
\hline Parity Median(min-max) & $1(0-1)$ & $0(0-1)$ & $1(0-2)$ & $2(1-4)$ & 0.000 \\
\hline Progesteron (ng/ml) & $9.98 \pm 4.61$ & $10.38 \pm 5.51$ & $5.95 \pm 3.32$ & $8.19 \pm 3.92$ & 0.006 \\
\hline
\end{tabular}

Table 1. Demographic and baseline data of the group

Data are presented as mean \pm standard deviation, *; Kruskal Wallis test, BMI: Body Mass Index, Endo: Endometrioma; PCOS; Polycystic Ovary Syndrome, US: Unexplained Subfertility
There were statistically significant differences between fertile group and each ones of study groups as expected.

Table 2 shows the distribution of IGFBP - 1, PGE2 and OPN levels. All markers were statistically different within all groups. 


\begin{tabular}{|c|c|c|c|c|c|}
\hline & $\begin{array}{c}\text { PCOS } \\
(\mathbf{n = 2 4})\end{array}$ & $\begin{array}{c}\text { US } \\
(\mathbf{n = 2 5})\end{array}$ & $\begin{array}{c}\text { Endo } \\
(\mathbf{n = 1 7})\end{array}$ & $\begin{array}{c}\text { Control } \\
(\mathbf{n = 1 8})\end{array}$ & P-Value* \\
\hline IGFBP-1 $(\mathbf{n g} / \mathbf{m l})$ & $310.22 \pm 70.76$ & $396.51 \pm 130.55$ & $391.18 \pm 118.86$ & $377.36 \pm 123.10$ & $\mathbf{0 . 0 2 8}$ \\
\hline PGE2 $(\mathbf{n g} / \mathbf{m l})$ & $367.75 \pm 96.37$ & $292.68 \pm 123.42$ & $259.16 \pm 117.80$ & $239.25 \pm 106.97$ & $\mathbf{0 . 0 0 3} \dagger$ \\
\hline OPN $(\mathbf{n g} / \mathbf{m l})$ & $12.09 \pm 7.72$ & $13.03 \pm 9.61$ & $16.67 \pm 6.27$ & $10.04 \pm 4.74$ & $\mathbf{0 . 0 2 9}$ \\
\hline
\end{tabular}

Table 2. The distribution of IGFBP - 1, PGE2 and OPN

Data are presented as mean \pm standard deviation, Endo; Endometrioma, IGFBP-1; insulin-like growth factor binding protein 1, PCOS; Polycystic Ovary Syndrome, PGE2; prostoglandin E2, OPN; osteopontin, US; Unexplained Subfertility, *; Kruskal Wallis test, †; ovulatory PCOS vs control group

The comparison of IGFBP - 1, PGE2 and OPN levels between study groups and control group is given in Table 3.

\begin{tabular}{|l|l|l|l|}
\hline & $\begin{array}{l}\text { PCOS vs Control } \\
\text { (p value) }\end{array}$ & $\begin{array}{l}\text { US vs Control } \\
\text { (p value) }\end{array}$ & $\begin{array}{l}\text { Endo } \\
\text { Control } \\
\text { (p value) }\end{array}$ \\
\hline $\begin{array}{l}\text { IGFBP }-\mathbf{1} \\
(\mathbf{n g} / \mathbf{m l})\end{array}$ & 0.349 & 1.000 & 1.000 \\
\hline $\begin{array}{l}\text { PGE2 } \\
(\mathbf{n g} / \mathbf{m l})\end{array}$ & $\mathbf{0 . 0 0 2}$ & 0.769 & 1.000 \\
\hline $\begin{array}{l}\text { OPN } \\
(\mathbf{n g} / \mathbf{m l})\end{array}$ & 1.000 & 1.000 & 0.068 \\
\hline
\end{tabular}

Table 3. The comparison of IGFBP-1, PGE2 and OPN levels between the two groups

Endo; Endometrioma, IGFBP-1; insulin-like growth factor binding protein 1, PGE2; prostoglandin E2, OPN; osteopontin, PCOS; Polycystic Ovary Syndrome, US: Unexplained Subfertility, *; Mann Whitney U test

Within all markers, only PGE2 levels were higher in ovulatory PCOS group (367.75 \pm 96.37$)$ compared to control group (239.25 \pm 106.97$)$. The difference was statistically significant (0.002).

\section{Discussion}

In this prospective cross-sectional study, our aim was to compare the levels of IGFBP-1, osteopontin and PGE2 in the endometrial washing fluids of healthy, fertile women and patients with ovulatory PCOS, endometrioma and unexplained subfertility during implantation window. PGE2 levels were significantly higher in the ovulatory PCOS patients than in the control group.

There is increasing evidence regarding the irregular expression of uterine receptivity markers in the endometrium of women with ovulatory PCOS. Navarra et al [17] determined significantly high PGE2 levels in polycystic ovaries. In another study, it was reported that in patients receiving in vitro fertilization and ovum donation, PGE2 levels in the endometrial fluid increased substantially during the implantation window [16]. They reported that 24 hours prior to embryo transfer, PGE2 levels might predict pregnancy results and thus, PGE2 might be the potential non-invasive biomarker of endometrial receptivity. These findings are similar to the results of our study. Elevated PGE2 in ovulatory PCOS accompanies reduced a tendency to apoptosis. This has been observed by Ryu et al [18] in the endometrial cells. It is known that cells over secreting COX-2 have inability to increase proliferation and the ability to escape from apoptosis. The Gap 1 phase of this cell cycle is prolonged [19]. In addition to the basics of physiopathology appearing to be the increased apoptosis inhibition in the endometrial cells, PGE2 may contribute to endometrial dysfunction with its effects on cell proliferation, angiogenesis and immunosuppression by affecting the estrogen levels.

Low IGFBP-1 was shown in PCOS and obesity, but there were some inconsistencies in the literature. In the study of Kelly et al [20], IGFBP-1 levels were detected significantly lower in the ovulatory PCOS group compared to the control group. In obese PCOS patients, IGFBP-1 was lower than the normal weight patients with ovulatory PCOS. No significant difference was observed between obese PCOS-obese control groups and normal-weight control groups with PCOS. Reduced IGFBP-1 in ovulatory PCOS was related to ovarian hyperandrogenism mechanism. BMI was determined by Kelly et al [20] as the main determinant of serum IGFBP-1. Considering that the BMI of ovulatory PCOS group was high $(28.6 \mathrm{~kg} / \mathrm{m} 2)$, it may explain the low levels of IGFBP-1 in our study, even though it was not statistically significant. In a recent study; mid-luteal OPN levels indicated similar distributions in ovulatory PCOS phenotype patients compared to controls [21]. A significant reduction was observed in OPN levels in infertile women with isolated PCO. However, in this study ovulatory dysfunction was the main reason for subfertility. The contradictory results affect the comparability of this data compared to our study.

For the endometrioma group of our study, IGFBP-1, OPN and PGE2 levels in mud-luteal phase were similar to the control group. It is known that genetic factors are related to the development and progression of endometriosis; however, genes related to endometriosis are not identified. It is believed that IGFBPs play an important role in cell apoptosis, proliferation and pathophysiology of endometriosis. In one study, it was determined that IGFBP-1 was not associated with endometriosis whereas IGFBP-3 was significantly associated with endometriosis [22]. $\alpha v \beta 3$ integrin and osteopontin, the extracellular matrix ligand thereof, play a role in the regulation of endometrial receptivity. While osteopontin expression was not affected in women with endometriosis, it was shown that $\alpha v \beta 3$ integrin expression was reduced. Interestingly, when $\alpha v \beta 3$ expression is lacking, OPN's binding to the surface epithelium is quite limited. This evidence indicates that the endometrium of some women with endometriosis is dysfunctional and responsible for the decrease in fecundity [23]. In our study, relative elevation in OPN and IGFBP-1 levels 
Gibson DA, Simitsidellis I, Kelepouri O, et al. (2018) Dehydroepiandrosterone enhances decidualization in women of advanced reproductive age. Fertil Steril. 109(4):728-734.

may affect negatively both the inhibition
implantation in the implantation window.

In our study, in the unexplained subfertility group, IGFBP-1, OPN and PGE2 levels in the midluteal phase were similar to the control group. Studies investigating unexplained subfertility and endometrial dysfunction in the implantation window are limited in literature. Recently, osteopontin and its receptor $\alpha \mathrm{v} \beta 3$ integrin were suggested as important complexes in embryo implantation and therefore they may be useful as endometrial receptivity markers. In one study, no statistically significant differences were found in terms of $\alpha v \beta 3$ integrin or osteopontin expression. Even though both glycoprotein concentrations were high in the 8th post-ovulation day, a significant lack in co-expressions during the implantation window period was observed. In a popular study Casals et al [14] concluded that the complex of $\alpha v \beta 3$ and OPN was functional in endometrial receptivity and implantation.

The strengths of our study were that the markers studied were diverse and the numbers of subjects were sufficient. However, the weaknesses of our study were that the diagnosis of endometrioma was made by imaging methods; the control group consisted of coincidental, relatively older fertile women, owing to consecutive recruitment. The limitations of our study were lack of the power analysis. Therefore; although midluteal PGE2 expression was higher in the endometrioma patients and unexplained subfertility groups compared to the control group, the difference was not statistically significant.

In conclusion; ovulatory PCOS, unexplained subfertility and endometriosis were related to the disrupted endometrium receptivity in the literature. PGE2 might be an indicator of a reduced endometrial receptivity that might be responsible for the low pregnancy rates in ovulatory PCOS patients according to results of our study. Because a single biomarker that each of many genes expresses is not sufficient in explaining the implantation bio mechanism and high numbers of biomarkers play a role in endometrial receptivity, there is a need for a more extensive studies comprising a high number of markers for the endometrium receptivity in different infertility issues.

\section{Disclosure statement:}

No potential conflict of interest was reported by the authors.

\section{References}

1. Paria BC, Reese J, Das SK, et al. (2002) Deciphering the crosstalk of implantation: advances and challenges. Science. 296:2185-8.

2. Kao LC, Tulac S, Lobo S, et al. (2002) Global gene profiling in human endometrium during the window of implantation. Endocrinology. 143:2119-38.

3. Strowitzki T, Germeyer A, Popovici R, et al. (2006) The human endometrium as a fertility-determining factor. Hum Reprod Update. 12:617-30.

4. Lee J, Oh J, Choi E, et al. (2007) Differentially expressed genes implicated in unexplained recurrent spontaneous abortion. Int $\mathrm{J}$ Biochem Cell Biol. 39;2265-77.

5. Aghajanova L, Hamilton AE, Giudice LC. (2008) Uterine receptivity to human embryonic implantation: histology, biomarkers, and transcriptomics. Semin Cell Dev Biol. 19(2);204-11.

6. Wang HS, Chard T. (1999) IGFs and IGF-binding proteins in the regulation of human ovarian and endometrial function. $\mathrm{J}$ Endocrinol. 161(1):1-13.
8. Tamura I, Jozaki K, Sato S, et al. (2018) The distal upstream region of insulin-like growth factor-binding protein-1 enhances its expression in endometrial stromal cells during decidualization. J Biol Chem. 293(14):5270-5280.

9. Apparao KBC, Murray MJ, Fritz MA, et al. (2001) Osteopontin and its receptor a $B$, integrin are coexpressed in the human endometrium during ihe menstrual cycle but regulated differentially. $\mathrm{J}$ of Clinical Endocrinology and Metabolism. $86: 4491-3000$.

10. Lessey BA. (2002) Adhesion molecules and implantation. J Reprod Immunol. 55:101-12.

11. Von Wolff M, Strowitzki T, Becker V, et al. (2001) Endometrial osteopontin, a ligand of beta3-integrin, is maximally expressed around the time of the "implantation window". Fertility and Sterility. 76:775-81.

12. Johnson GA, Burghardt RC, Bazer FW, et al. (2003) Osteopontin: roles in implantation and placent at ton. Biology of Reproduction. 69:1458-71.

13. Makker A, Singh MM. (2006) Endometrial receptivity: clinical assessment in relation to fertility, infertility, and antinfertility. Medicinal Research Reviews. 26:699-746.

14. Casals G, Ordi J, Creus M, et al. (2008) Osteopontin and alphavbeta3 integrin expression in the endometrium of infertile and fertile women. Reprod Biomed Online. 16(6):808-16.

15. Wang XB, Qi QR, Wu KL, et al. (2018) Role of osteopontin in decidualization and pregnancy success. Reproduction. 155(5):423-432.

16. Vilella F, Ramirez L, Berlanga O, et al. (2013) PGE2 and PGF2 $\alpha$ concentrations in human endometrial fluid as biomarkers for embryonic implantation. J Clin Endocrinol Metab. 98(10):412332.

17. Navarra P, Andreani CL, Lazzarin N, et al. (1996) Increased production and release of prostaglandin-E2 by human granulosa cells from polycystic ovaries. Prostaglandins. 52(3):187-97.

18. Ryu HS, Chang KH, Yang HW, et al.(2000) High cyclooxygenase-2 expression in stage IB cervical cancer with lymph node metastasis or parametrial invasion. Gynecol Oncol.

19. DuBois RN, Shao J, Tsujii M, et al. (1996) G1 delay in cells overexpressing prostaglandin endoperoxide synthase-2. Cancer Res. 56:733-7.

20. Kelly CJ, Stenton SR, Lashen H. (2011) Insulin-like growth factor binding protein-1 in PCOS: a systematic review and metaanalysis. Hum Reprod Update. 17(1):4-16.

21. DuQuesnay R, Wright C, Aziz AA, et al. (2009) Infertile women with isolated polycystic ovaries are deficient in endometrial expression of osteopontin but not alphavbeta3 integrin during the implantation window. Fertil Steril. 91(2):489-99.

22. Kim H, Ku SY, Kim SH, et al. (2012) Association between endometriosis and polymorphisms in insulin-like growth factor binding protein genes in Korean women. Eur J Obstet Gynecol Reprod Biol. 162(1):96-101.

23. Lessey BA. Implantation defects in infertile women with endometriosis. Ann N Y Acad Sci. 2002;955:265-280. 76:320-5. 